\title{
Contemporary Issues in the Field
}

By Jack M. Gorman, MD

Although most issues of CNS Spectrums showcase several articles around a specific theme, we also receive an ever-increasing number of outstanding unsolicited manuscripts each month. All manuscripts, unsolicited or not, must endure a rigorous peer-review process to ensure we are offering our readers the best possible features. Articles in CNS Spectrums are increasingly being cited in other Index Medicus peer-reviewed journals and at international conferences. This month, instead of publishing articles on a particular topic, we decided to present several recently accepted and important unsolicited articles. Hence, we offer you an interesting mixture of original data and reviews discussing timely issues in the worlds of psychiatry and neurology.

Robert G. Bota, MD, and colleagues tackle one of the emerging questions in schizophrenia research: How early in the course of the illness should we intervene? They look at records obtained during the prodromal phase of schizophrenia, a period that can last for several years, during which patients who ultimately exhibit the classical positive symptoms of the illness first begin to manifest social withdrawal, cognitive problems, odd behavior and ideas, and mood swings. The researchers found that patients with schizophrenia who met the full diagnostic criteria for any Axis I illness during the prodromal phase are more likely to require hospitalization once frank psychosis begins. They also found that antipsychotic treatment during the prodrome significantly reduced hospitalization. This article adds to a growing understanding of the prognostic importance of the prodromal period of schizophrenia and raises the possibility that early intervention with antipsychotics may moderate the severity of the illness.

Turning to the important area of transcultural psychiatry, S. Shalom Feinberg, MD, presents a fascinating account of the considerations necessary when prescribing psychotropic drugs to members of the Orthodox Jewish community. I have often lamented that psychiatrists, particularly residents and junior practitioners, believe it is "politically correct" when taking a psychiatric history to ignore the possibility that ethnicity, nationality, culture, and religion may play roles in shaping an individual's personality and behavior. Feinberg makes clear that failing to account for specific religious rules in the case of Orthodox Jews may make effective treatment impossible. Psychiatry's traditional hostility to religion-Freud called it "mass- delusion" in Civilization and Its Discontents-is fortunately beginning to weaken, making articles like this one helpful for clinicians. Similar articles concerning other ethnic, national, and religious groups that may be similarly informative are most welcome.

Salvatore Gentile, MD, tackles the elusive neurobiology of postpartum psychiatric disorders and points out that $10 \%$ to $20 \%$ of new mothers may develop psychiatric problems, and one logical theory points the finger at changes in maternal estrogen levels. Thus far it has been difficult to prove this theory, however, Gentile offers ideas on how studies could be conducted to offer us more useful information. He considers whether there is sufficient evidence to warrant estrogen replacement therapy for postpartum depression and other psychiatric disorders. This is a complex question, given the bevy of concerns that have been raised concerning estrogen replacement therapy for postmenopausal women and Gentile offers reasonable guidelines for clinicians in determining whether this strategy is indicated.

Eric Hollander, MD, and colleagues note that although pharmacotherapy and psychotherapy for obsessive-compulsive disorder (OCD) have improved recently, many patients remain either undertreated or entirely refractory to treatment. For them, a variety of experimental brain stimulation techniques may provide relief. Although none have yet been approved by the Food and Drug Administration specifically for OCD (vagus nerve stimulation has been approved for the treatment of refractory depression), several have been tested in OCD patients with promising results.

A case report is presented detailing magnetic resonance imaging changes in a patient with migraine headache and transient global amnesia following cardiac angiography. Both psychiatrists and neurologists will enjoy the train of events in this report.

This will be my final column as a faculty member of Mount Sinai School of Medicine. Next month, I move to Boston to become president and psychiatrist-in-chief of McLean Hospital, chair of Partners Psychiatry and Mental Health, and Professor of Psychiatry at Harvard Medical School. Mount Sinai was the birthplace of CNS Spectrums and will continue to play an integral part in its intellectual and academic life. I want to thank all of my Mount Sinai colleagues for their help and support in bringing you CNS Spectrums each month. CNS 